Pacific Journal of Mathematics

GROTHENDIECK LOCALLY CONVEX SPACES OF
CONTINUOUS VECTOR VALUED FUNCTIONS 


\title{
GROTHENDIECK LOCALLY CONVEX SPACES OF CONTINUOUS VECTOR VALUED FUNCTIONS
}

\author{
Francisco J. Freniche
}

\begin{abstract}
Let $\mathscr{C}(X, E)$ be the space of continuous functions from the completely regular Hausdorff space $X$ into the Hausdorff locally convex space $E$, endowed with the compact-open topology. Our aim is to characterize the $\mathscr{C}(X, E)$ spaces which have the following property: weak-star and weak sequential convergences coincide in the equicontinuous subsets of $\mathscr{C}(X, E)^{\prime}$. These spaces are here called Grothendieck spaces. It is shown that in the equicontinuous subsets of $E^{\prime}$ the $\sigma\left(E^{\prime}, E\right)$ and $\beta\left(E^{\prime}, E\right)$-sequential convergences coincide, if $\mathscr{C}(X, E)$ is a Grothendieck space and $X$ contains an infinite compact subset. Conversely, if $X$ is a $G$-space and $E$ is a strict inductive limit of Fréchet-Montel spaces $\mathscr{C}(X, E)$ is a Grothendieck space. Therefore, it is proved that if $E$ is a separable Fréchet space, then $E$ is a Montel space if and only if there is an infinite compact Hausdorff $X$ such that $\mathscr{C}(X, E)$ is a Grothendieck space.
\end{abstract}

1. Introduction. In this paper $X$ will always denote a completely regular Hausdorff topological space, $E$ a Hausdorff locally convex space, and $\mathscr{C}(X, E)$ the space of continuous functions from $X$ into $E$, endowed with the compact-open topology. When $E$ is the scalar field of reals or complex numbers, we write $\mathscr{C}(X)$ instead $\mathscr{C}(X, E)$.

It is well known that $\mathscr{C}(X, E)$ is a Montel space whenever $\mathscr{C}(X)$ and $E$ so are, hence, if and only if $X$ is discrete and $E$ is a Montel space (see [5], [16]).

We study what happens when $X$ has the following weaker property: the compact subsets of $X$ are $G$-spaces (see below for definitions).

We obtain in Theorem 4.4 that if $E$ is a Fréchet-Montel space and $X$ has that property, then $\mathscr{C}(X, E)$ is a Grothendieck locally convex space. The key in the proof is the following fact: every countable equicontinuous subset of $\mathscr{C}(X, E)^{\prime}$ lies, via a Radon-Nikodým theorem, in a suitable $L^{1}\left(\tau, E_{\beta}^{\prime}\right)$. As a consequence of a theorem of Mújica [10], the same result is true when $E$ is a strict inductive limit of Fréchet-Montel spaces.

In $\S 3$ we study the converse of 4.4 . In Corollary 3.3 it is proved that if $X$ contains an infinite compact subset, $E$ is a Fréchet separable space and $\mathscr{C}(X, E)$ is a Grothendieck space, then $E$ is a Montel space. This property characterizes the Montel spaces among the Fréchet separable spaces. 
Finally, in $\S 5$ we study the Grothendieck property in $\mathscr{B}(\Sigma, E)$, the space of $\Sigma$-totally measurable functions, by using the results for $\mathscr{C}(X, E)$.

2. Generalities. A compact Hausdorff topological space $K$ is called a $G$-space whenever $\mathscr{C}(K)$ is a Grothendieck Banach space, i.e. the weak-star and weak sequential convergences coincide in $\mathscr{C}(K)^{\prime}$ [6].

We extend here this concept to completely regular spaces.

2.1. Definition. $X$ is a $G$-space if every compact subset $K$ of $X$ is a $G$-space.

If $X$ is compact, both definitions coincide [6]. Let us remark that there exist non-compact non-discrete $G$-spaces. Indeed, the topological subspace of the Stone-Čech compactification of a countable discrete set obtained removing a cluster point, is such a space.

We introduce a new definition of Grothendieck locally convex space, so that $\mathscr{C}(X)$ is a Grothendieck space if and only if $X$ is a $G$-space.

2.2. Definition. $E$ is a Grothendieck space whenever the $\sigma\left(E^{\prime}, E\right)$ and $\sigma\left(E^{\prime}, E^{\prime \prime}\right)$-sequential convergences coincide in the equicontinuous subsets of $E^{\prime}$.

In [17] the $T G$-spaces are defined as those spaces $E$ in which the $\sigma\left(E^{\prime}, E\right)$ - and $\sigma\left(E^{\prime}, E^{\prime \prime}\right)$-sequential convergences coincide. When one deals with $\mathscr{C}(X)$ spaces, our definition seems to be more reasonable than that of [17] (see 2.4 and 2.5).

The following permanence properties of the class of Grothendieck locally convex spaces are easy to see, thus we state them without proof.

2.3. Proposition. (a) E is a Grothendieck space if and only if every, or some, dense subspace of $E$ so is.

(b) Let $T: E \rightarrow F$ be a linear continuous operator such that for every bounded subset $B$ of $F$ there is a bounded subset $C$ of $E$ so that $B$ is contained in the closure of $T(C)$. Then $F$ is a Grothendieck space if $E$ so is.

(c) If $E$ is the inductive limit of the sequence $\left(E_{n}\right)$ of Grothendieck spaces, and if every bounded subset of $E$ is contained in some $E_{n}$, then $E$ is a Grothendieck space.

2.4. TheOREM. $\mathscr{C}(X, E)$ is a Grothendieck space if and only if $\mathscr{C}(K, E)$ so is for every compact subset $K$ of $X$. In particular, $X$ is a $G$-space if and only if $\mathscr{C}(X)$ is a Grothendieck space. 
Proof. Let us recall that, if $K$ is a compact subset of $X$, the restriction map $T$ is a continuous linear operator from $\mathscr{C}(X, E)$ into $\mathscr{C}(K, E)$.

If $B \subset \mathscr{C}(K, E)$ is bounded, then the bounded subset $C$ of $\mathscr{C}(X, E)$, whose elements $g$ can be written $g=\sum_{n \leq m} f_{n}(\cdot) e_{n}$ with $f_{n} \in \mathscr{C}(X), 0 \leq f_{n}$ $\leq 1, \sum_{n \leq m} f_{n} \leq 1$, and $e_{n} \in \bigcup\{h(K): h \in B\}$, satisfies $\overline{T(C)} \supset B$ (see $[14$, I.5.3]).

If $\mathscr{C}(X, E)$ is a Grothendieck space, $\mathscr{C}(K, E)$ so is by $2.3(\mathrm{~b})$.

Conversely, let $\left(g_{n}^{\prime}\right)$ be an equicontinuous and $\sigma\left(\mathscr{C}(X, E)^{\prime}, \mathscr{C}(X, E)\right)$-null sequence. By [14, III.3 and III.4], there exist a compact subset $K$ of $X$ and an equicontinuous sequence $\left(h_{n}^{\prime}\right)$ in $\mathscr{C}(K, E)^{\prime}$ such that $g_{n}^{\prime}=h_{n}^{\prime} \circ T$ for all $n \in \mathbf{N}$. Since $\left(h_{n}^{\prime}\right)$ is $\sigma\left(\mathscr{C}(K, E)^{\prime}\right.$, $T(\mathscr{C}(K, E)))$-null and equicontinuous, it is also $\sigma\left(\mathscr{C}(K, E)^{\prime}\right.$, $\left.\mathscr{C}(K, E)^{\prime \prime}\right)$-null if $\mathscr{C}(K, E)$ is a Grothendieck space. It follows that $\left(g_{n}^{\prime}\right)$ is $\sigma\left(\mathscr{C}(X, E)^{\prime}, \mathscr{C}(X, E)^{\prime \prime}\right)$-null.

2.5. REMARK. We use an example of Haydon [4] to show that, while in the class of barrelled spaces the $T G$-spaces and the Grothendieck spaces do coincide, this is not true in general.

Choose, for each infinite sequence in $\mathbf{N}$, a cluster point in the Stone-Čech compactification of $\mathbf{N}$, and let $X$ be the topological subspace of that compactification, formed by $\mathbf{N}$ and these cluster points. Then every compact subset of $X$ is finite, $\mathscr{C}(X)$ is infrabarrelled and every $f \in \mathscr{C}(X)$ is bounded. By Theorem $2.4, X$ is a $G$-space. Let $f_{n}^{\prime}(f)=$ $n^{-1} f(n)$ for all $f \in \mathscr{C}(X)$ and $n \in \mathbf{N}$. Then $\left(f_{n}^{\prime}\right)$ is a $\sigma\left(\mathscr{C}(X)^{\prime}, \mathscr{C}(X)\right)$-null sequence in $\mathscr{C}(X)^{\prime}$, that is not $\sigma\left(\mathscr{C}(X)^{\prime}, \mathscr{C}(X)^{\prime \prime}\right)$-null because it is not equicontinuous.

3. Necessary conditions for $\mathscr{C}(X, E)$ to be a Grothendieck space. It is well known, and easy to see, that $\mathscr{C}(X)$ and $E$ are topologically isomorphic to complemented subspaces of $\mathscr{C}(X, E)$. By 2.3(b), $\mathscr{C}(X)$ and $E$ must be Grothendieck spaces if $\mathscr{C}(X, E)$ is such a space.

However, unless $X$ is pseudofinite, i.e. their compact subsets are finite (hence $\mathscr{C}(X, E)$ is a Grothendieck space if and only if $E$ so is, by Theorem 2.4), $E$ has a stronger property if $\mathscr{C}(X, E)$ is a Grothendieck space, as we prove in the next theorem. To prove it we recall the following result of [2]:

Theorem A. Let $E$ and $F$ be Hausdorff locally convex spaces, and suppose that $F$ contains a subspace topologically isomorphic to the subspace of $c_{0}$ whose elements have only finitely many non-zero coordinates.

If the injective tensor product $F \otimes_{\varepsilon} E$ is a Grothendieck space, then the $\sigma\left(E^{\prime}, E\right)$ - and $\beta\left(E^{\prime}, E\right)$-sequential convergences coincide in the equicontinuous subsets of $E^{\prime}$. 
As was noted in [2], if $X$ is not pseudofinite, then $\mathscr{C}(X)$ contains a subspace topologically isomorphic to the above mentioned subspace of $c_{0}$. Moreover, the injective tensor product $\mathscr{C}(X) \otimes_{\varepsilon} E$ can be linear and topologically identified with a dense subspace of $\mathscr{C}(X, E)$, namely, the subspace of all finite dimensional valued elements of $\mathscr{C}(X, E)$. Thus we obtain from Theorem A and Proposition 2.3 (a):

3.1. Theorem. If $\mathscr{C}(X, E)$ is a Grothendieck space and $X$ contains an infinite compact subset, then the $\sigma\left(E^{\prime}, E\right)$ - and $\beta\left(E^{\prime}, E\right)$-sequential convergences coincide in the equicontinuous subsets of $E^{\prime}$.

3.2. Remark. By Theorem 2.4, if $X$ is pseudofinite and $E$ is a Grothendieck Banach space, $\mathscr{C}(X, E)$ is a Grothendieck space. However, if $E$ is infinite dimensional, the conclusion of Theorem 3.1 does not hold [11].

Using Theorem 3.1 and $[7,11.6 .2]$, we obtain the following corollary, converse of Theorem 4.4:

3.3. COROLlaRY. If $E$ is a Fréchet separable space, $X$ is not pseudofinite and $\mathscr{C}(X, E)$ is a Grothendieck space, then $E$ is a Montel space.

3.4. Remark. It is unknown for us if Corollary 3.3 is true without the separability assumption on $E$. This is related with the following question raised in [7, pg. 247]: is a Fréchet space $E$ already a Montel space if every $\sigma\left(E^{\prime}, E\right)$-convergent sequence in $E^{\prime}$ converges for $\beta\left(E^{\prime}, E\right)$ ?

4. Sufficient conditions for $\mathscr{C}(X, E)$ to be a Grothendieck space. We shall need some facts about vector integration, many of those can be found in [1] and [15].

Let $(X, \Sigma, \tau)$ be a complete measure space with $\tau(X) \leq 1$. We denote by $\mathscr{S}(\Sigma, E)$ (resp. $\mathscr{B}(\Sigma, E), L^{1}(\tau, E), L^{\infty}(\tau, E)$ ) the vector space of $\Sigma$-simple (resp. $\Sigma$-totally measurable, $\tau$-integrable, $\tau$-essentially bounded) $E$-valued (classes of) functions. Recall that $\mathscr{S}(\Sigma, E)$ and $\mathscr{B}(\Sigma, E)$ are endowed with the uniform convergence topology, and that the topology of $L^{1}(\tau, E)$ is defined by the seminorms $u \rightarrow \int p(u(x)) d \tau(x)$, where $p$ runs over the set of all continuous seminorms in $E$ (unless contrary specification, all integrals will be extended to $X$ ).

The following Radon-Nikodým theorem is proved in [1]:

THEOREM B. If $E$ is a quasi-complete (CM)-space, $\mu: \Sigma \rightarrow E$ is a countably additive vector measure, of bounded variation and $\tau$-absolutely continuous, then there exists $u \in L^{1}(\tau, E)$ such that $\mu(A)=\int_{A} u(x) d \tau(x)$ for every $A \in \Sigma$. 
Let us recall that $E$ is a quasi-complete (CM)-space, if, for instance, it is either a Fréchet-Montel space or a (DF)-Montel space [1].

Firstly we extend the classical duality theorem $L^{1}-L^{\infty}$ to $L^{1}\left(\tau, E_{\beta}^{\prime}\right)$, where $E$ is a Fréchet-Montel space.

The following lemma can be easily proved. As usual, $p_{L}$ will denote the gauge of the absolutely convex set $L$ in its linear span.

4.1. LeMMA. If $u \in \mathscr{S}\left(\Sigma, E^{\prime}\right)$, namely, $u=\sum_{i \leq m} \chi_{A_{i}} e_{i}^{\prime}$ with $\left(A_{i}\right)_{i \leq m}$ disjoint in $\Sigma$, then

$$
\int p_{B^{0}}(u(x)) d \tau(x) \leq \tau\left(\bigcup_{i \leq m} A_{i}\right) \sup _{i \leq m} p_{B^{0}}\left(e_{i}^{\prime}\right)
$$

for every bounded subset $B$ of $E$.

4.2. THEOREM. Let $E$ be a Fréchet-Montel space. The relation

$$
u^{\prime}(u)=\int u(x)(v(x)) d \tau(x) \text { for all } u \in L^{1}\left(\tau, E_{\beta}^{\prime}\right)
$$

defined for $u^{\prime} \in L^{1}\left(\tau, E_{\beta}^{\prime}\right)^{\prime}$ and $v \in L^{\infty}(\tau, E)$, is an algebraic isomorphism between $L^{1}\left(\tau, E_{\beta}^{\prime}\right)^{\prime}$ and $L^{\infty}(\tau, E)$.

Proof. Let $v \in L^{\infty}(\tau, E)$. The map $x \rightarrow u(x)(v(x))$ is measurable for every $u \in L^{1}\left(\tau, E_{\beta}^{\prime}\right)$, because $v$ is strongly measurable and the assertion is clearly true when $v \in \mathscr{S}(\Sigma, E)$.

Furthermore, if $Z \in \Sigma$ is a $\tau$-null set such that $B=v(S \backslash Z)$ is bounded, then we have

$$
|u(x)(v(x))| \leq p_{B^{0}}(u(x))
$$

for every $x \in X \backslash Z$.

Hence $x \rightarrow u(x)(v(x))$ is $\tau$-integrable, and we can define a linear form $u^{\prime}$ on $L^{1}\left(\tau, E_{\beta}^{\prime}\right)$ by (1). Moreover, it follows from (2) that $u^{\prime}$ is continuous.

Conversely, fix $u^{\prime} \in L^{1}\left(\tau, E_{\beta}^{\prime}\right)^{\prime}$. There exists a bounded subset $B$ of $E$ such that

$$
\int p_{B^{0}}(u(x)) d \tau(x) \leq 1 \text { implies }\left|u^{\prime}(u)\right| \leq 1
$$

for every $u \in L^{1}\left(\tau, E_{\beta}^{\prime}\right)$.

We define a map $\mu: \Sigma \rightarrow E^{\prime \prime}$ by

$$
\mu(A)\left(e^{\prime}\right)=u^{\prime}\left(\chi_{A} e^{\prime}\right)
$$

for every $A \in \Sigma$ and $e^{\prime} \in E^{\prime}$ (it follows easily from Lemma 4.1 and (3) that $\left.\mu(A) \in E^{\prime \prime}\right)$. Since $E$ is reflexive we can suppose that $\mu(A) \in E$. 
Clearly, $\mu: \Sigma \rightarrow E$ is a finitely additive vector measure. We shall show that $\mu$ is countably additive: let $A$ be the union of the disjoint sequence $\left(A_{n}\right)$ in $\Sigma$. Given an absolutely convex zero-neighborhood $U$ in $E$ and $\varepsilon>0$, we choodse $\lambda$ with $0<\lambda<\infty$ such that $B \subset \lambda U$, and $m_{0} \in \mathbf{N}$ such that $\lambda \tau\left(\cup_{n>m} A_{n}\right) \leq \varepsilon$ for every $m \geq m_{0}$. Since

$$
e^{\prime}(\mu(A))-\sum_{n \leq m} e^{\prime}\left(\mu\left(A_{n}\right)\right)=u^{\prime}\left(\chi_{\cup_{n>m} A_{n}} e^{\prime}\right)
$$

it follows from Lemma 4.1 and (3) that

$$
\left|e^{\prime}(\mu(A))-\sum_{n \leq m} e^{\prime}\left(\mu\left(A_{n}\right)\right)\right| \leq \varepsilon
$$

for every $m \geq m_{0}$ and $e^{\prime} \in U^{0}$, as desired.

Furthermore, if $A=\bigcup_{n \leq m} A_{n}$ where $\left(A_{n}\right)_{n \leq m}$ is disjoint in $\Sigma$, and if $\varepsilon>0$, there exists $\left(e_{n}^{\prime}\right)_{n \leq m}$ in $U^{0}$ such that

$$
\sum_{n \leq m} p_{U}\left(\mu\left(A_{n}\right)\right) \leq \sum_{n \leq m} e_{n}^{\prime}\left(\mu\left(A_{n}\right)\right)+\varepsilon=u^{\prime}\left(\sum_{n \leq m} \chi_{A_{n}} e_{n}^{\prime}\right)+\varepsilon
$$

Hence the $p_{U}$-variation of $\mu$ satisfies the inequality $V_{p_{U}} \mu(A) \leq \lambda \tau(A)$, from Lemma 4.1 and (3) again.

Thus $\mu$ is $\tau$-absolutely continuous and has bounded variation. By Theorem B, there exists $v \in L^{1}(\tau, E)$ such that

$$
\mu(A)=\int_{A} v(x) d \tau(x) \text { for every } A \in \Sigma .
$$

We claim that $v$ is $\tau$-essentially bounded and satisfies (1). Indeed, let $\left(U_{j}\right)_{j}$ be a countable basis in $E$ of absolutely convex zero-neighborhoods. Choose, for each $j \in \mathbf{N}, \lambda_{j}$ such that $0<\lambda_{j}<\infty$ and $B \subset \lambda_{j} U_{j}$.

By Lemma 4.1, (3), (4) and (5), we have

$$
\left|\int_{A} e^{\prime}(v(x)) d \tau(x)\right| \leq \lambda_{j} \tau(A)
$$

for all $e^{\prime} \in U_{j}^{0}, A \in \Sigma$ and $j \in \mathbf{N}$.

Let $\left(e_{j, k}^{\prime}\right)_{k}$ be a sequence in $U_{j}^{0}$ such that $p_{U_{j}}(e)=\sup _{k}\left|e_{j, k}^{\prime}(e)\right|$ for every $e \in E$.

By (6), there exists $Z \in \Sigma$ with $\tau(Z)=0$ such that $\left|e_{j, k}^{\prime}(v(x))\right| \leq \lambda_{j}$ for all $x \in X \backslash Z$ and all $j, k \in \mathbf{N}$. Hence $v(X \backslash Z)$ is bounded in $E$.

Finally, it follows from (4) that (1) is true for all $u \in \mathscr{S}\left(\Sigma, E^{\prime}\right)$, and, by density, for every $u \in L^{1}\left(\tau, E_{\beta}^{\prime}\right)$. This concludes the proof.

Assume that $X$ is compact Hausdorff and $\Sigma$ contains the Borel subsets of $X$. For each $u \in L^{1}\left(\tau, E_{\beta}^{\prime}\right)$, denote by $\nu_{u}$ the vector measure of density $u$ with respect to $\tau$. If $p$ is a continuous seminorm in $E$, the subset 
$F$ of $L^{1}\left(\tau, E_{\beta}^{\prime}\right)$ defined by the condition $V_{p} \nu_{u}(X)<\infty$, is a linear subspace. If $u \in F$ then $v_{u}$ has bounded semivariation, thus it defines a continuous linear form on $\mathscr{S}(\Sigma, E)$, which extends by continuity to the whole space $\mathscr{B}(\Sigma, E)$ [15]. Let $T u \in \mathscr{C}(X, E)^{\prime}$ be the restriction to $\mathscr{C}(X, E)$ of this linear form, i.e.

$$
(T u)(g)=\int g(x) d \nu_{u}(x)
$$

for every $g \in \mathscr{C}(X, E)$.

4.3. Lemma. The map $T: F \rightarrow \mathscr{C}(X, E)^{\prime}$ defined by (7) is a linear continuous operator, when $\mathscr{C}(X, E)^{\prime}$ is endowed with the strong topology with respect to $\mathscr{C}(X, E)$.

Proof. We have, for each $u \in F$,

$$
(T u)(g)=\int u(x)(g(x)) d \tau(x)
$$

for every $g \in \mathscr{C}(X, E)$. Indeed, the dominated convergence theorem and a standard density argument show that it suffices to see (8) when $g$ belongs to $\mathscr{S}(\Sigma, E)$, that is trivially true.

Let $H$ be a bounded subset of $\mathscr{C}(X, E)$. Then $B=\bigcup\{g(X): g \in H\}$ is a bounded subset of $E$. Hence, by $(8),|(T u)(g)| \leq \int p_{B^{0}}(u(x)) d \tau(x)$ and the lemma follows.

We are now ready to prove the sufficient condition:

4.4. THEOREM. Let $X$ be a completely regular Hausdorff $G$-space and $E$ a Fréchet-Montel space. Then $\mathscr{C}(X, E)$ is a Grothendieck space.

Proof. By 2.4 we can suppose, without loss of generality, that $X$ is compact.

Let $\left(g_{n}^{\prime}\right)_{n}$ be an equicontinuous sequence in $\mathscr{C}(X, E)^{\prime}$. By [14, III.4.5] there exists a continuous seminorm $p$ in $E$ such that $V_{p} \mu_{n}(X) \leq 1$, for every $n \in \mathbf{N}$, where $\mu_{n}$ is the representing measure of $g_{n}^{\prime}[14$, III $]$.

Let $\tau=\sum_{n} 2^{-n} V_{p} \mu_{n} . \tau$ is a countably additive $[0,1]$-valued Borel measure, by [14, III.2.5]. Let $\Sigma$ be the completed $\sigma$-field of the Borel field of $X$ with respect to $\tau$. We shall denote also by $\tau$ and $\mu_{n}$ the natural extensions of the earlier measures to $\Sigma$. 
Since $E$ is a Montel space, the measure $\mu_{n}: \Sigma \rightarrow E_{\beta}^{\prime}$ is countably additive. Clearly $V_{p} \mu_{n} \leq 2^{n}$, thus $\mu_{n}$ has bounded variation and is $\tau$-absolutely continuous (when it is considered as an $E_{\beta}^{\prime}$-valued measure).

We apply Theorem B, obtaining, for each $n \in \mathbf{N}$, a function $u_{n} \in$ $L^{1}\left(\tau, E_{\beta}^{\prime}\right)$ such that $\mu_{n}$ is the vector measure of density $u_{n}$ with respect to $\tau$.

Clearly $u_{n} \in F$ and $T u_{n}=g_{n}^{\prime}$, for every $n \in \mathbf{N}$.

Fix $g^{\prime \prime} \in \mathscr{C}(X, E)^{\prime \prime}$. By Lemma 4.3 and Theorem 4.2, there exists $v \in L^{\infty}(\tau, E)$ such that $g^{\prime \prime}\left(g_{n}^{\prime}\right)=\int u_{n}(x)(v(x)) d \tau(x)$ for every $n \in \mathbf{N}$.

Let $Z$ be a set in $\Sigma$ with $\tau(Z)=0$ and $v(X \backslash Z)$ bounded. The function $v_{1}=\chi_{X \backslash Z} v$ is totally measurable, because $E$ is Montel and metrizable.

Given $\varepsilon>0$, we can choose $v_{2} \in \mathscr{S}(\Sigma, E)$ such that $p\left(v_{3}(x)\right) \leq \varepsilon / 2$, for every $x \in X$, if $v_{3}=v_{1}-v_{2}$. Hence,

$$
\left|\int u_{n}(x)\left(v_{3}(x)\right) d \tau(x)\right|=\left|\int v_{3}(x) d \mu_{n}(x)\right| \leq \varepsilon / 2
$$

for every $n \in \mathbf{N}$, because $V_{p} \mu_{n}(X) \leq 1$.

On the other hand, if $\left(g_{n}^{\prime}\right)$ is $\sigma\left(\mathscr{C}(X, E)^{\prime}, \mathscr{C}(X, E)\right)$-null, then $\left(\mu_{n}(A)(e)\right)$ is a null sequence, for every $e \in E$ and $A \in \Sigma$. Indeed, since $X$ is a $G$-space, for each $e \in E$, the weak-star null sequence $\left(\mu_{n}(\cdot)(e)\right)$ in $\mathscr{C}(X)^{\prime}$, is also weak null, hence $\left(\mu_{n}(A)(e)\right)$ is null for every Borel subset $A$ of $X$, and so for every $A \in \Sigma$.

Since $v_{2}$ is simple, it follows that

$$
\lim _{n \rightarrow \infty} \int u_{n}(x)\left(v_{2}(x)\right) d \tau(x)=0 .
$$

By (9) and (10), $\left(g^{\prime \prime}\left(g_{n}^{\prime}\right)\right)$ is a null sequence, and we have shown that $\left(g_{n}^{\prime}\right)$ is $\sigma\left(\mathscr{C}(X, E)^{\prime}, \mathscr{C}(X, E)^{\prime \prime}\right)$-null.

4.5. Corollary. Let $X$ be a completely regular Hausdorff G-space and $E$ the inductive limit of the sequence $\left(E_{n}\right)$ of Fréchet-Montel spaces, such that every bounded subset of $E$ is localized in some $E_{n}$. Then $\mathscr{C}(X, E)$ is a Grothendieck space.

Proof. We can again suppose $X$ compact. By [10], the inductive limit of the sequence $\left(\mathscr{C}\left(X, E_{n}\right)\right)$ is a dense topological subspace of $\mathscr{C}(X, E)$. By Proposition 2.3 (a) and (c), and Theorem 4.4, it follows that $\mathscr{C}(X, E)$ is a Grothendieck space. 
4.6. Corollary. Let $E$ be a Fréchet separable space. The following conditions are equivalent:

(a) $E$ is a Montel space.

(b) There exists a non-pseudofinite completely regular Hausdorff space $X$ such that $\mathscr{C}(X, E)$ is a Grothendieck space.

(c) For every completely regular Hausdorff $G$-space $X, \mathscr{C}(X, E)$ is a Grothendieck space.

Proof. Use 4.4 and 3.3.

5. Application to spaces of totally measurable functions. Let $X$ be a nonempty set and $\Sigma$ a field of subsets of $X$. We will say that a subset $B$ of $X$ is open if for every $x \in B$ there is $A \in \Sigma$ with $x \in A$ and $A \subset B$. Endowed $X$ with this topology, let $X^{*}$ be the Hausdorff space associated to $X, \pi: X \rightarrow X^{*}$ the quotient map, and $\Sigma^{*}=\{\pi(A): A \in \Sigma\}$.

The following lemma is easily established:

5.1. LeMma (a) $X^{*}$ is a completely regular Hausdorff zero-dimensional topological space.

(b) The map $A \in \Sigma \rightarrow \pi(A) \in \Sigma^{*}$ is a Boolean isomorphism.

(c) The map $g \in \mathscr{B}\left(\Sigma^{*}, E\right) \rightarrow g \circ \pi \in \mathscr{B}(\Sigma, E)$ is a topological isomorphism, and its restriction to $\mathscr{S}\left(\Sigma^{*}, E\right)$ so is onto $\mathscr{S}(\Sigma, E)$.

(d) The map $x^{*} \in X^{*} \rightarrow\left\{B^{*} \in \Sigma^{*}: x^{*} \in B^{*}\right\} \in \mathscr{P}\left(\Sigma^{*}\right)$ is one-toone.

By using 5.1, when one studies the linear topological properties of $\mathscr{B}(\Sigma, E)$, it can be supposed that $X$ is a dense subspace of a Hausdorff compact zero-dimensional topological space $K$ (namely, the Stone space of the Boolean algebra $\Sigma$ ), and $\Sigma$ is the trace in $X$ of the Boolean algebra of open and closed subsets of $K$. In this context we have the following theorem:

5.2. THEOREM. There exists a subspace of $\mathscr{B}(\Sigma, E)$, containing $\mathscr{S}(\Sigma, E)$, that is topologically isomorphic to $\mathscr{C}(K, E)$.

Proof. It is easy to check that the set of restrictions to $X$ of all elements of $\mathscr{C}(K, E)$ is such a subspace.

By Proposition $2.3($ a), it follows that $\mathscr{B}(\Sigma, E)$ is a Grothendieck space if and only if $\mathscr{C}(K, E)$ so is. Hence we can apply to $\mathscr{B}(\Sigma, E)$ the results of $\S \S 3$ and 4 . 
5.3. REMARK. The question of when $\mathscr{B}(\Sigma)$ (equivalently, $\mathscr{C}(K)$ ) is a Grothendieck space is related to the validity of the Vitali-Hahn-Saks theorem for finitely additive scalar measures on $\Sigma$, of bounded variation. For instance, if $\Sigma$ is $\sigma$-complete, or more generally, $\Sigma$ has the subsequential interpolation property, then $\mathscr{B}(\Sigma)$ is a Grothendieck space (see [13] and [3]).

Finally, we show that the following result of Mendoza [8], can be easily deduced from their earlier results in [9] and our Theorem 5.2.

5.4. Theorem. Suppose $\Sigma$ infinite. Then $\mathscr{B}(\Sigma, E)$ is infrabarrelled (resp. barrelled) if and only if $E_{\beta}^{\prime}$ has property (B) of Pietsch [12, 1.5.8], and $E$ is infrabarrelled (resp. barrelled).

Proof. Let us observe that $\mathscr{S}(\Sigma, E)$ is a large dense subspace of $\mathscr{B}(\Sigma, E)$. Indeed, if $H$ is a bounded subset of $\mathscr{B}(\Sigma, E)$, then the set of all $g$ in $\mathscr{S}(\Sigma, E)$ for which there exists $h \in H$ with $g(X) \subset h(X)$, is a bounded subset of $\mathscr{S}(\Sigma, E)$ whose closure in $\mathscr{B}(\Sigma, E)$ contains $H$.

Thus Theorem 5.2 implies that $\mathscr{B}(\Sigma, E)$ is infrabarrelled whenever $\mathscr{C}(K, E)$ so is, hence we have the first equivalence of the theorem, by [9].

If $\mathscr{B}(\Sigma, E)$ is barrelled, then $E$ is barrelled and $\mathscr{B}(\Sigma, E)$ is infrabarrelled, so $E_{\beta}^{\prime}$ has property (B). The converse follows easily because $\mathscr{C}(K, E)$ is topologically isomorphic to a dense subspace of $\mathscr{B}(\Sigma, E)$, by 5.2 .

5.5. REMARK. We have also shown in 5.4 that, if $\Sigma$ is infinite, $\mathscr{S}(\Sigma, E)$ is infrabarrelled if and only if $E_{\beta}^{\prime}$ has property $(B)$ and $E$ is infrabarrelled, a result of Mendoza [8]. In [2] we prove that $\mathscr{S}(\Sigma, E)$ is barrelled if and only if $\mathscr{S}(\Sigma)$ and $E$ so are, and $E$ is nuclear.

\section{REFERENCES}

[1] G. Y. H. Chi, On the Radon-Nikodym theorem and locally convex spaces with the Radon-Nikodym property, Proc. Amer. Math. Soc., 61 (1977), 245-253.

[2] F. J. Freniche, Barrelledness of the space of vector valued and simple functions, Math. Ann., 267 (1984), 479-486.

[3] - The Vitali-Hahn-Saks theorem for Boolean algebras with the subsequential interpolation property, Proc. Amer. Math. Soc., 92 (1984), 362-366.

[4] R. Haydon, Trois exemples dans la théorie des espaces de fonctions continues, C. R. Acad. Sci. Paris, 276A, (1973), 685-687.

[5] R. Hollstein, Permanence properties of $\mathscr{C}(X, E)$, Manuscripta Math., 38 (1982), 41-58. 
[6] R. Isbell and Z. Semadeni, Projections constants and spaces of continuous functions, Trans. Amer. Math. Soc., 107 (1963), 38-48.

[7] H. Jarchow, Locally Convex Spaces, Stuttgart, B. G. Teubner 1981.

[8] J. Mendoza, Barrelledness conditions on $\mathscr{S}(\Sigma, E)$ and $\mathscr{B}(\Sigma, E)$, Math. Ann., 261 (1982), 11-22.

[9] Necessary and sufficient conditions for $\mathrm{C}(X, E)$ to be barrelled or infrabarrelled, Simon Stevin, 57 (1983), 103-123.

[10] J. Mújica, Spaces of continuous functions with values in an inductive limit, Lecture Notes in Pures and Applied Math., 83 (1983).

[11] A. Nissenzweig, $w^{*}$-sequential convergence, Israel J. Math., 22 (1975), 266-272.

[12] A. Pietsch, Nuclear Locally Convex Spaces, Berlin-Heidelberg-New York, Springer 1972.

[13] W. Schachermayer, On some classical measure-theoretic theorems for non-sigma-complete Boolean algebras, Johannes Kepler Universität Linz, Linz-Auhof 1980.

[14] J. Schmets, Spaces of Vector-Valued Continuous Functions, Lecture Notes in Math. 1003, Berlin-Heidelberg-New York-Tokyo, Springer 1983.

[15] A. H. Suchat, Integral representations theorems in topological vector spaces, Trans. Amer. Math. Soc., 172 (1972), 373-397.

[16] S. Warner, The topology of compact convergence on continuous functions spaces, Duke Math. J., 25 (1958), 265-282.

[17] A. Wilansky, Modern Methods in Topological Vector Spaces, New York, McGraw-Hill 1978.

Received May 10, 1984.

UNIVERSIDAD DE SEVILLA

C / TARFIA SN.

SEVILLA-12

EspaÑA 



\section{PACIFIC JOURNAL OF MATHEMATICS EDITORS}

\author{
V. S. VARADARAJAN (Managing Editor) \\ University of California \\ Los Angeles, CA 90024 \\ Charles R. DePrima \\ California Institute of Technology \\ Pasadena, CA 91125 \\ R. FINN \\ Stanford University \\ Stanford, CA 94305
}

\author{
HeRmanN FlaschKa \\ University of Arizona \\ Tucson, AZ 857.21
}

RAMESH A. GANGOLli

University of Washington

Seattle, WA 98195

ROBION KIRBY

University of California

Berkeley, CA 94720
C. C. MOORE

University of California

Berkeley, CA 94720

H. SAMELSON

Stanford University

Stanford, CA 94305

HAROLD STARK

University of California, San Diego

La Jolla, CA 92093

\section{ASSOCIATE EDITORS}
R. ARENS
E. F. BECKENBACH
B. H. NeumanN
F. WOLF
K. YoSHIDA
(1906-1982)

\section{SUPPORTING INSTITUTIONS}

\begin{abstract}
UNIVERSITY OF ARIZONA
UNIVERSITY OF BRITISH COLUMBIA

CALIFORNIA INSTITUTE OF TECHNOLOGY

UNIVERSITY OF CALIFORNIA

MONTANA STATE UNIVERSITY

UNIVERSITY OF NEVADA, RENO

NEW MEXICO STATE UNIVERSITY

OREGON STATE UNIVERSITY
\end{abstract}

\author{
UNIVERSITY OF OREGON \\ UNIVERSITY OF SOUTHERN CALIFORNIA \\ STANFORD UNIVERSITY \\ UNIVERSITY OF HAWAII \\ UNIVERSITY OF TOKYO \\ UNIVERSITY OF UTAH \\ WASHINGTON STATE UNIVERSITY \\ UNIVERSITY OF WASHINGTON
}

The Supporting Institutions listed above contribute to the cost of publication of this Journal, but they are not owners or publishers and have no responsibility for its content or policies.

Mathematical papers intended for publication in the Pacific Journal of Mathematics should be in typed form or offset-reproduced (not dittoed), double spaced with large margins. Please do not use built up fractions in the text of the manuscript. However, you may use them in the displayed equations. Underline Greek letters in red, German in green, and script in blue. The first paragraph must be capable of being used separately as a synopsis of the entire paper. In particular it should contain no bibliographic references. Please propose a heading for the odd numbered pages of less than 35 characters. Manuscripts, in triplicate, may be sent to any one of the editors. Please classify according to the scheme of Math. Reviews, Index to Vol. 39. Supply name and address of author to whom proofs should be sent. All other communications should be addressed to the managing editor, or Elaine Barth, University of California, Los Angeles, California 90024.

There are page-charges associated with articles appearing in the Pacific Journal of Mathematics. These charges are expected to be paid by the author's University, Government Agency or Company. If the author or authors do not have access to such Institutional support these charges are waived. Single authors will receive 50 free reprints; joint authors will receive a total of 100 free reprints. Additional copies may be obtained at cost in multiples of 50 .

The Pacific Journal of Mathematics is issued monthly as of January 1966. Regular subscription rate: $\$ 190.00$ a year (5 Vols., 10 issues). Special rate: $\$ 66.00$ a year to individual members of supporting institutions.

Subscriptions, orders for numbers issued in the last three calendar years, and changes of address should be sent to Pacific Journal of Mathematics, P.O. Box 969, Carmel Valley, CA 93924, U.S.A. Old back numbers obtainable from Kraus Periodicals Co., Route 100, Millwood, NY 10546.

The Pacific Journal of Mathematics at P.O. Box 969, Carmel Valley, CA 93924 (ISSN 0030-8730) publishes 5 volumes per year. Application to mail at Second-class postage rates is pending at Carmel Valley, California, and additional mailing offices. Postmaster: Send address changes to Pacific Journal of Mathematics, P.O. Box 969, Carmel Valley, CA 93924.

PUBLISHED BY PACIFIC JOURNAL OF MATHEMATICS, A NON-PROFIT CORPORATION

Copyright $\odot 1985$ by Pacific Journal of Mathematics 


\section{Pacific Journal of Mathematics}

Vol. 120, No. $2 \quad$ October, 1985

Philip Marshall Anselone and Mike Treuden, Regular operator

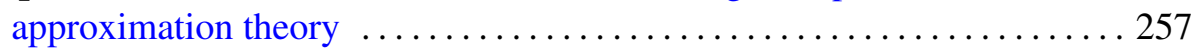

Giuseppe Baccella, Semiprime $\aleph-Q F 3$ rings $\ldots \ldots \ldots \ldots \ldots \ldots \ldots \ldots . \ldots \ldots$

Earl Robert Berkson and Thomas Alastair Gillespie, The generalized M.

Riesz theorem and transference $\ldots \ldots \ldots \ldots \ldots \ldots \ldots \ldots . \ldots 279$

Joachim Boidol, A Galois-correspondence for general locally compact

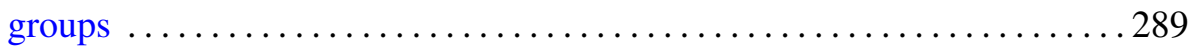

Joseph Eugene D'Atri, Josef Dorfmeister and Yan Da Zhao, The isotropy

representation for homogeneous Siegel domains ............... 295

C. Debiève, On Banach spaces having a Radon-Nikodým dual

Michael Aaron Freedman, Existence of strong solutions to singular

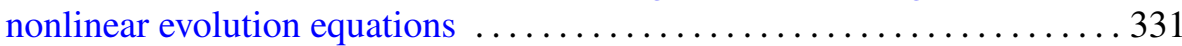

Francisco Jose Freniche, Grothendieck locally convex spaces of continuous

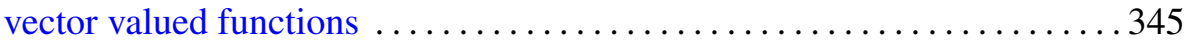

Hans-Peter Künzi and Peter Fletcher, Extension properties induced by complete quasi-uniformities . ............................ 357

Takaŝi Kusano, Charles Andrew Swanson and Hiroyuki Usami, Pairs of

positive solutions of quasilinear elliptic equations in exterior domains . . 385

Angel Rafael Larotonda and Ignacio Zalduendo, Spectral sets as Banach

manifolds

J. Martínez-Maurica and C. Pérez García, A new approach to the

Kreı̆n-Milman theorem

Christian Pommerenke, On the boundary continuity of conformal maps . . . 423

M. V. Subba Rao, Some Rogers-Ramanujan type partition theorems

Stephen Edwin Wilson, Bicontactual regular maps .........

Jaap C. S. P. van der Woude, Characterizations of (H)PI extensions

Kichoon Yang, Deformation of submanifolds of real projective space

Subhashis Nag, Errata: "On the holomorphy of maps from a complex to a

real manifold" 